\title{
Éclosions de rougeole au sein d'une population ne pratiquant pas la vaccination, Alberta 2013
}

\author{
Kershaw $\mathrm{T}^{1^{*}}$, Suttorp $\mathrm{V}^{2}$, Simmonds $\mathrm{K}^{3}$ et St. Jean $\mathrm{T}^{3}$ \\ Programme canadien d'épidémiologie de terrain, Agence de la santé publique du Canada, Edmonton (Alberta) \\ Population and Public Health Division, Alberta Health Services, Lethbridge (Alberta) \\ 3 Surveillance and Assessment Branch, ministère de la Santé de l'Alberta, Edmonton (Alberta) \\ Auteur-ressource : tanis.kershaw@gov.ab.ca
}

\section{Résumé}

Contexte : Une éclosion de rougeole a été déclarée dans le sud de l'Alberta le 18 octobre 2013, à la suite du signalement au service local de santé publique d'un cas survenu chez un adolescent non vacciné ayant voyagé récemment aux Pays-Bas. L'adolescent avait eu des contacts avec un grand nombre de personnes non vaccinées alors qu'elles étaient contagieuses. Par conséquent, le risque de propagation était élevé. Le risque d'une éclosion de rougeole dans ce secteur avait été reconnu par le médecin hygiéniste responsable de la zone sud et la planification en vue d'une éclosion avait été amorcée en août 2013.

Méthodes : Plusieurs mesures de santé publique ont été mises en œuvre pour maîtriser l'éclosion : une campagne de vaccination de masse; une dose de vaccin adaptée aux éclosions de rougeole, rubéole et oreillons (RRO) chez les nourrissons de 6 à 12 mois; des communications dans les collectivités touchées et avoisinantes; une ligne d'urgence consacrée à la rougeole; une équipe mobile d'évaluation de la rougeole; ainsi qu'un centre d'évaluation de la rougeole.

Résultats : Au total, 42 cas confirmés ont été répertoriés au cours de l'éclosion entre le 16 octobre et le 25 novembre. Un peu plus de la moitié des cas étaient de sexe masculin $(52,4 \%)$. L'âge moyen était de 12 ans (étendue : < 1 à 24 ans) et l'âge médian, de 13 ans. Un cas a été hospitalisé, mais aucun décès n'est survenu. Tous étaient non vaccinés. Les cas habitaient dans cinq collectivités à proximité immédiate de Lethbridge. Tous, sauf deux cas, avaient des liens épidémiologiques avec 10 ménages.

Conclusion : La planification effectuée avant l'éclosion a été déterminante dans le confinement de la propagation à 10 ménages. L'exploration de stratégies visant à accroître la couverture vaccinale des populations non vaccinées est essentielle pour prévenir de futures éclosions de rougeole. Lorsque l'acceptation de la vaccination n'est pas uniforme, d'autres stratégies de santé publique devraient être planifiées et mises en œuvre afin de prévenir une propagation plus importante.

\section{Introduction}

La rougeole est un virus très contagieux qui se transmet par voie aérienne avec un taux d'attaque secondaire de plus de $90 \%$ entre personnes réceptives (1). Dans environ $30 \%$ des cas, les personnes atteintes présentent une ou plusieurs complications et, dans les pays développés, la rougeole entraînera un décès dans 1 à 2 cas par 1000 habitants $(1,2)$. La rougeole est évitable par la vaccination : une dose unique de vaccin contenant le virus de la rougeole offre une efficacité de $85 \%$ à $95 \%$ et une seconde dose élève l'efficacité à près de $100 \%$ (1). En Alberta, le programme de vaccination des enfants contre la rougeole prévoit une dose à l'âge de 12 mois et une seconde dose entre 4 et 6 ans. En Alberta, les dernières éclosions importantes de rougeole sont survenues en 1997 (242 cas), en 1999 (17 cas) et en 2000 (123 cas).

La prestation des services de santé en Alberta (y compris les services de santé publique) est assurée par l'Alberta Health Services, qui se divise en cinq services régionaux. La région située au sud de Calgary, qui comprend Lethbridge et Medicine Hat, compte divers groupes culturels, dont bon nombre ne sont pas favorables 
à la vaccination et ont déjà connu des éclosions de maladies évitables par la vaccination. Dans le comté de Lethbridge, dans une région à proximité, située à l'ouest, au nord et à l'est de Lethbridge, la dernière éclosion de rougeole est survenue en 1997, ce qui fait en sorte qu'une large cohorte d'enfants nés après 1997 n'est pas immunisée contre la rougeole, par exposition naturelle ou par vaccination. Un important groupe démographique dans le comté de Lethbridge est composé de familles ayant des liens étroits avec les Pays-Bas, où, depuis mai 2013, une éclosion de rougeole à grande échelle a lieu au sein d'une communauté religieuse dont l'opposition à la vaccination était connue $(3,4)$. Le médecin hygiéniste responsable de la zone sud a reconnu le risque d'importation de la rougeole dans le sud de l'Alberta et a amorcé, en août 2013, la planification en vue d'une éclosion potentielle.

Cette phase de préparation à une éclosion de rougeole est fondée sur les principes d'urgence utilisés en gestion des catastrophes, notamment ceux du système de gestion des incidents. Au cours de cette période, un certain nombre de plans et de stratégies clés ont été élaborés pour la zone sud : 1) la mise en œuvre des directives de 2012 du Comité consultatif national de l'immunisation pour la vaccination des travailleurs de la santé contre la rougeole; 2) une vaste campagne de sensibilisation destinée aux intervenants externes et au public dans le but de conscientiser la population aux risques de rougeole, de promouvoir la vaccination et de l'informer au sujet des mesures à prendre pour réduire la transmission à ceux qui ne se font pas vacciner; 3) une entente entre les services de santé publique locaux et les chefs religieux, les médecins et les administrateurs scolaires; et 4) l'élaboration d'un plan de mise sur pied d'un centre d'évaluation de la rougeole, qui prévoit des outils de triage, la constitution d'une réserve et l'équipement des salles pour qu'elles répondent aux normes de pression négative de l'Association canadienne de normalisation.

Cette planification s'est avérée précieuse, car, le 16 octobre 2013, les services de santé publique locaux ont été informés d'un cas suspect de rougeole dans le comté de Lethbridge survenu chez un adolescent non vacciné ayant voyagé récemment aux Pays-Bas. II s'agissait d'un cas classique de rougeole, présentant un coryza et une toux (le 9 octobre), se poursuivant avec de la fièvre (le 11 octobre) et une éruption cutanée maculopapulaire (le 16 octobre). Grâce aux renseignements fournis aux familles au cours de la période de planification de la lutte contre la rougeole, la famille connaissait la nécessité de signaler les cas à la santé publique. Cette mesure a permis aux responsables de la santé publique d'obtenir des échantillons de laboratoire prélevés au domicile du client le 16 octobre et de réduire le risque d'exposition en milieu de soins de santé. Les résultats de laboratoire ont confirmé le 18 octobre qu'il s'agissait d'un cas de rougeole. La victime avait interagi, alors qu'elle était infectée, avec un grand nombre de personnes à un événement sportif public et à des événements religieux, scolaires et familiaux à l'occasion du congé de l'Action de grâce. La majorité de ces contacts s'étaient produits avec des membres d'une communauté religieuse qui n'est pas favorable à la vaccination. En tenant compte du risque de propagation dans une collectivité non vaccinée, une éclosion de rougeole a été déclarée dans la zone sud le 18 octobre 2013.

Dans le présent rapport, nous présentons les détails de cette éclosion, notamment son profil épidémiologique et les mesures de santé publique qui ont été mises en œuvre. Les enseignements tirés de cette expérience seront exposés afin que les autres collectivités publiques puissent en tenir compte lorsqu'elles auront à réagir en cas d'éclosion de rougeole, particulièrement auprès de populations qui ne se font pas vacciner.

\section{Méthodes}

L'éclosion de rougeole a été déclarée le 18 octobre 2013 et l'éradication a été confirmée le 6 janvier 2014. Des centres d'opérations d'urgence ont été ouverts dans la zone sud et dans les locaux de l'Alberta Health Services et du ministère de la Santé de l'Alberta. Les trois centres d'opérations d'urgence ont adhéré aux principes du système de gestion des incidents, ce qui a permis une collaboration et une communication fluides entre les organisations.

\section{Recherche des cas et activités de collecte de données}

La définition des cas de rougeole a été préalablement établie par le ministère de la Santé de l'Alberta dans les lignes directrices intitulées Public Health Notifiable Disease Management Guidelines (5). Une précision a été apportée à l'application de la définition de cas concernant le fait que la maladie clinique doit être identifiée par 
un professionnel de la santé, y compris de la santé publique, et ne doit pas être autodéclarée. Les définitions permettant d'identifier les cas confirmés, probables et suspects sont décrites au Tableau 1.

Tableau 1. Définitions des cas confirmés, probables et suspects de rougeole en Alberta

\begin{tabular}{|c|c|}
\hline Classification des cas & Définition \\
\hline Cas confirmé & $\begin{array}{l}\text { Confirmation de l'infection par une analyse en laboratoire, en l'absence d'une récente } \\
\text { immunisation par un vaccin contenant le virus de la rougeole, devant satisfaire à l'un des } \\
\text { critères suivants : } \\
\text { - Détection d'acide nucléique caractéristique du virus de la rougeole (p. ex. par réaction } \\
\text { de polymérisation en chaîne en temps réel) dans un échantillon clinique; } \\
\text { - Séroconversion ou augmentation marquée (par un facteur d'au moins quatre) du titre } \\
\text { des anticorps lgG spécifiques au virus de la rougeole dans le sérum en phase de } \\
\text { convalescence par rapport au sérum en phase aiguë, par une épreuve sérologique } \\
\text { standard; } \\
\text { - Sérologie positive pour les anticorps lgM spécifiques au virus de la rougeole chez une } \\
\text { personne ayant un lien épidémiologique avec un cas confirmé en laboratoire ou qui a } \\
\text { voyagé récemment dans une région où l'on sait qu'il y a des cas de rougeole; } \\
\text { - Isolement du virus de la rougeole dans un échantillon clinique (p. ex. écouvillonnage du } \\
\text { nasopharynx, urine); } \\
\text { - Maladie clinique* chez une personne qui a un lien épidémiologique avec un cas confirmé } \\
\text { en laboratoire. }\end{array}$ \\
\hline Cas probable & $\begin{array}{l}\text { Maladie clinique satisfaisant à l'un des critères suivants : } \\
\text { - En l'absence d'enquêtes en laboratoire appropriées et d'un lien épidémiologique avec un } \\
\text { cas confirmé en laboratoire; } \\
\text { - Chez une personne qui a voyagé récemment dans une région où l'on sait qu'il y a des } \\
\text { cas de rougeole. }\end{array}$ \\
\hline Cas suspect & $\begin{array}{l}\text { Maladie clinique même lorsque l'éruption cutanée maculopapulaire est présente depuis } \\
\text { moins de trois jours. }\end{array}$ \\
\hline
\end{tabular}

La recherche de contacts a été faite auprès de toutes les relations du cas index et des cas subséquents. Toutefois, en raison des événements publics auxquels le cas index a participé, il y a eu également une exposition importante dans la collectivité. Par conséquent, les médias ont publié des communiqués dans la zone sud et dans l'ensemble de la province pour diffuser de l'information sur la rougeole auprès du public. Une ligne téléphonique a été mise en place spécialement pour répondre aux demandes de renseignements supplémentaires. Tous les cas suspects ont fait l'objet d'une enquête de santé publique et, une fois confirmés, ont été consignés dans un formulaire de signalement des maladies à déclaration obligatoire et entrés dans la base de données Communicable Disease and Outbreak Management.

\section{Analyse épidémiologique}

Une épidémiologie descriptive des cas a été réalisée en continu. L'analyse du réseau social des cas et des membres de leur foyer a été effectuée pour 10 familles ayant des liens épidémiologiques entre elles à l'aide du logiciel Pajek. Enfin, pour chaque cas, la liste des contacts familiaux a été extraite de la base de données de gestion des cas et comportait leurs antécédents à l'égard de la vaccination ou de la contraction de la maladie. En outre, une liste des contacts étroits avec les cas a été extraite afin permettre de visualiser les relations connues des cas dans le réseau. Le taux d'attaque global des ménages et celui spécifique aux ménages non vaccinés ont été calculés pour les familles ayant des liens épidémiologiques entre elles. Cinq personnes ont été exclues de ces deux calculs de taux d'attaque : le cas index, le cas coprimaire (cas probable), une personne qui 
a refusé de poursuivre le suivi de santé publique (la nature du cas n'a pu être confirmée) et deux personnes qui avaient reçu deux doses de vaccin contenant le virus de la rougeole. Vingt-et-une personnes ont été exclues du calcul du taux d'attaque chez les personnes non vaccinées : vingt d'entre elles avaient des antécédents autodéclarés de la maladie et l'une avait reçu une dose de vaccin contenant le virus de la rougeole.

\section{Méthodes de laboratoire}

Le laboratoire provincial de santé publique de l'Alberta a effectué des analyses moléculaires de réaction de polymérisation en chaîne en temps réel (PCR en temps réel) sur des écouvillonnages du nasopharynx et des échantillons d'urine, et a mené des tests sérologiques dirigés vers les anticorps spécifiques au virus de la rougeole sur des échantillons sanguins. Les échantillons dont le résultat des analyses PCR était positif pour la rougeole étaient redirigés au Laboratoire national de microbiologie à Winnipeg aux fins de confirmation des résultats et de génotypage du virus. La confirmation sérologique était fondée sur la détection d'anticorps IgM spécifiques au virus de la rougeole dans un échantillon de sang prélevé en phase aiguë ou sur la séroconversion d'anticorps IgG spécifiques au virus de la rougeole entre un échantillon prélevé en phase aiguë par rapport à un autre prélevé en phase de convalescence.

\section{Mesures de santé publique}

Les équipes d'intervention en cas d'éclosion ont réagi rapidement et efficacement afin de mettre en œuvre les principales mesures de santé publique qui, pour la plupart, avaient été prévues au cours de la phase préparatoire, afin d'endiguer l'éclosion de rougeole. Les principales mesures comprenaient :

La communication : une communication en temps opportun, transparente, cohérente et fréquente avec les intervenants internes et externes, au moyen des médias sociaux et traditionnels, a été essentielle tout au long de l'éclosion. Dès la confirmation du cas index, le médecin hygiéniste local a immédiatement rencontré les membres du clergé et les pasteurs, ainsi que les administrateurs scolaires, pour les informer et les encourager à mettre en œuvre des mesures visant à réduire les risques de propagation de la maladie.

Une ligne d'urgence consacrée à la rougeole : Une ligne téléphonique accessible 24 heures sur 24, 7 jours sur 7, a été créée et mise en service durant l'éclosion pour traiter les demandes de renseignements du public. Tous les appels téléphoniques à propos de la rougeole reçus par Health Link, le service téléphonique provincial offrant aux Albertains des conseils gratuits donnés par des infirmières et de l'information sur la santé, étaient redirigés vers la ligne d'urgence. Celle-ci était également mise à la disposition des médecins pour qu'ils communiquent directement les renseignements de triage à l'équipe mobile d'évaluation de la rougeole.

Une équipe mobile d'évaluation de la rougeole : L'équipe mobile d'évaluation de la rougeole a été créée dans le but de donner accès à une évaluation 24 heures sur 24, 7 jours sur 7 , des cas éventuels de rougeole au sein de la collectivité et de tenir cette population à l'écart du milieu de soins de santé. Les équipes, composées de membres du personnel paramédical et des soins infirmiers à domicile en consultation par téléphone avec des médecins des services d'urgence, se rendaient au domicile des cas suspects afin d'examiner les personnes y vivant, de recueillir des échantillons de laboratoire et de donner des directives d'autogestion des soins, au besoin.

Un centre d'évaluation de la rougeole : Le centre d'évaluation de la rougeole a été mis en place à l'extérieur du service des urgences de l'hôpital régional et logeait dans une tente mobile d'isolement et de confinement, où se déroulaient l'évaluation et le traitement des patients atteints de la rougeole. Afin de réduire la probabilité de transmission de la rougeole dans le milieu de soins de santé, et en raison de la disponibilité limitée de salles en pression négative, la tente mobile d'isolement et de confinement s'est révélée être une mesure essentielle de santé publique.

Une campagne de vaccination et des doses de vaccin adaptées aux éclosions: Huit séances publiques de vaccination de masse ont été offertes dans la zone sud et une dose de vaccin RRO adaptée aux éclosions était administrée aux nourrissons de 6 à 12 mois dans les cliniques pédiatriques et à l'occasion des séances de vaccination de masse. 
La mise en quarantaine et l'exclusion : Les relations des cas qui étaient non vaccinées ont été encouragées à s'absenter de l'école ou du travail jusqu'à 21 jours après la détection du dernier cas dans leur ménage. Les cas ont été incités à se soumettre à la mise en quarantaine à domicile jusqu'à ce qu'ils ne soient plus considérés comme contagieux. Les avis de mise en quarantaine étaient émis uniquement sur demande, habituellement pour des raisons liées au travail.

\section{Résultats}

\section{Épidémiologie descriptive}

Au total, 43 cas (42 cas confirmés et 1 cas probable) ont été recensés au cours de l'éclosion. L'apparition de l'éruption cutanée est survenue chez le cas index le 16 octobre, et chez le dernier cas, le 25 novembre

(Figure 1).

Figure 1. Cas confirmé de rougeole en Alberta en fonction de la date d'apparition de l'éruption cutanée, du 16 octobre au 25 novembre 2013 ( $n=42)$

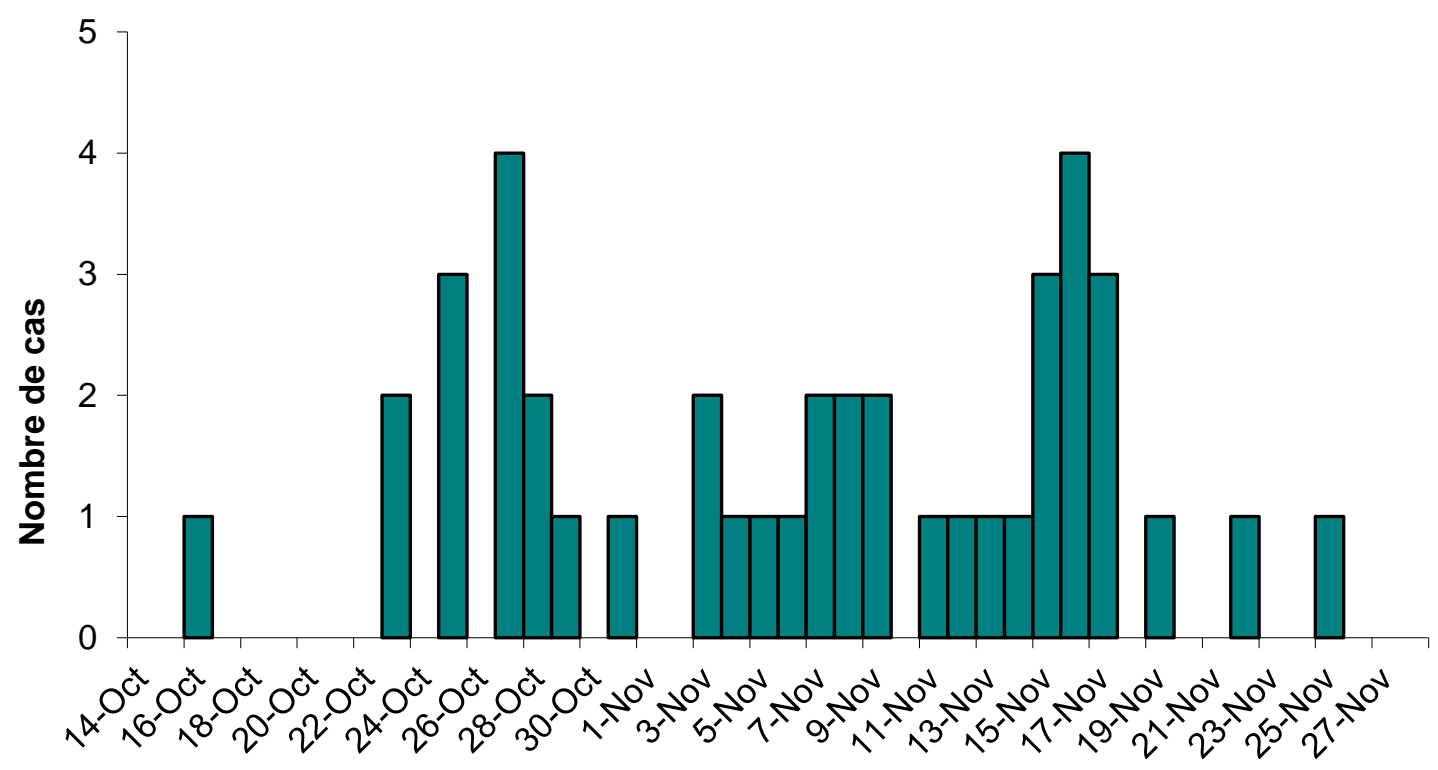

\section{Date de l'apparrition de l'éruption cutanée}

Un peu plus de la moitié des cas confirmés étaient de sexe masculin (52,4\%). L'âge moyen était de 12 ans (étendue : < 1 à 24 ans) et l'âge médian, de 13 ans. Six complications ont été signalées : deux cas de pneumonie, trois cas de déshydratation, et un cas d'otite moyenne. L'un des cas de pneumonie a nécessité une hospitalisation. Aucun décès n'a été associé à cette éclosion. Les cas habitaient dans cinq collectivités à proximité immédiate de Lethbridge. Tous étaient non vaccinés.

Tous, sauf deux cas, étaient concentrés dans 10 ménages. Ces deux derniers cas n'avaient aucun lien épidémiologique direct entre eux : l'un résidait dans une collectivité où il y avait des cas de rougeole et l'autre, dans une collectivité voisine exempte d'autres cas. La relation entre les cas et leurs contacts familiaux est illustrée à la Figure 2. Dans le diagramme, chaque grand cercle noir représente un ménage. Sept des dix ménages ont été en contact direct avec le cas index. 
Figure 2. Diagramme du réseau social des cas de rougeole et de leurs contacts familiaux dans la province de l'Alberta en 2013 ( $n=67)$

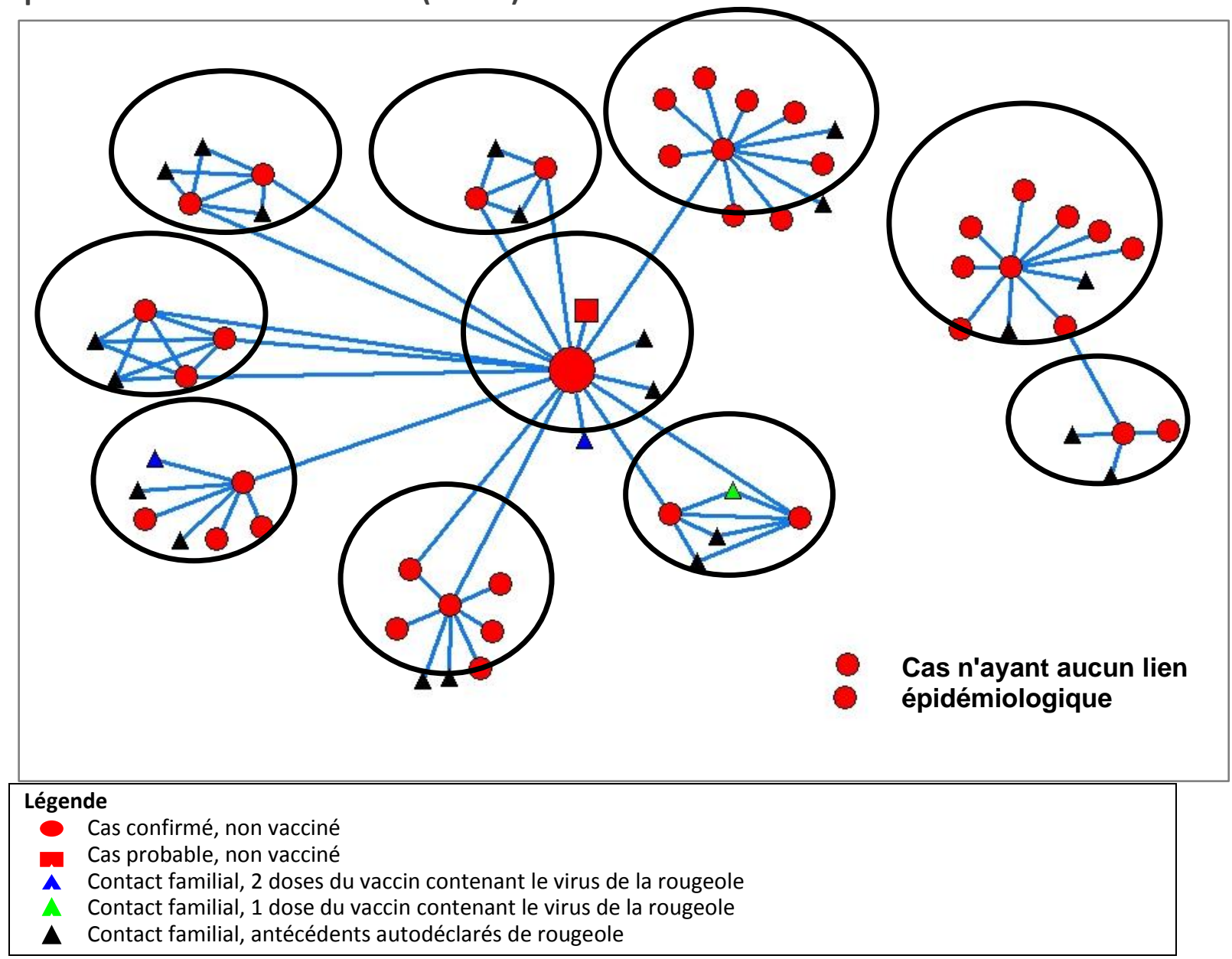

Le taux d'attaque global des ménages était de $65 \%$, mais celui des ménages non vaccinés était de $100 \%$. En d'autres termes, toutes les personnes dans ces 10 ménages, qui n'avaient pas reçu le vaccin contenant le virus de la rougeole et qui n'avaient pas d'antécédent autodéclaré de la maladie, ont contracté la rougeole.

\section{Résultats de laboratoire}

Seize des 42 cas confirmés (38\%) l'ont été par des analyses de laboratoire. Les 16 échantillons ont été envoyés aux fins de génotypage, dont 15 ont fait l'objet d'un génotypage. Tous les échantillons contenaient le génotype $\mathrm{D} 8$ de la rougeole (identique à la variante de la séquence MVs/Taunton.GBR/27,12), la même variante de la séquence identifiée dans l'éclosion en cours aux Pays-Bas (4) et observée en Angleterre en 2013 (6).

\section{Résultat des mesures de santé publique}

Entre le 21 octobre et le 3 janvier, les services de santé publique ont reçu un total de 7857 appels concernant la rougeole. Ceux-ci n'incluent pas les appels faits directement au médecin hygiéniste local ou à ligne d'urgence consacrée à la rougeole, car ceux-ci n'ont pas toujours été localisés. L'équipe mobile d'évaluation de la rougeole a été mobilisée 84 fois durant l'éclosion et 167 personnes ont été évaluées au centre d'évaluation de la rougeole. Au total, 1302 personnes ont été vaccinées au cours des 8 séances de vaccination.

\section{Discussion}

Des cas importés de rougeole surviennent chaque année au Canada, mais leur propagation secondaire est habituellement restreinte (7). C'est ce qui s'est produit en Alberta, de façon générale, car il s'agissait de la 
première éclosion de rougeole à grande échelle depuis 2000. En raison du lien direct du cas index avec une importante population de personnes réceptives, la propagation à partir ce de cas était prévisible. Toutefois, une planification rigoureuse avec les intervenants, la déclaration immédiate d'éclosion de rougeole ainsi que la confirmation du cas index a eu pour effet de limiter la durée de la transmission à environ six semaines. Bien que le nombre de 42 cas confirmés soit tout de même considéré comme important pour une éclosion de rougeole, il convient de noter que les cas étaient concentrés dans 10 ménages.

Cette éclosion a toutefois présenté quelques difficultés. Premièrement, les services de santé publique avaient eu connaissance de quelques cas non déclarés. Cependant, en raison des liens étroits entre la santé publique et les collectivités, la sous-déclaration des cas a été minime. Deuxièmement, les taux d'attaque n'ont pas pu être calculés pour tous les contacts en raison de la nature des événements publics auxquels a participé le cas index. Enfin, étant donné les ressources nécessaires pour gérer l'éclosion, les données de certaines mesures de santé publique n'ont pas toujours fait l'objet d'un suivi systématique et les données présentées sont donc sousestimées.

En dépit de ces difficultés, cette éclosion de rougeole était plus restreinte, moins grave et d'une durée plus courte que les autres éclosions de rougeole rencontrées récemment dans des populations non vaccinées ou sous-vaccinées. En 2011, une vaste éclosion de rougeole, qui a touché la province de Québec, a duré presque un an et a entraîné 776 cas, dont $11 \%$ ont dû être hospitalisés et $8 \%$ ont présenté des complications (8). De ce nombre, $79 \%$ étaient considérés comme non immuns (8). En date du 26 février 2014, plus de 2600 cas avaient été signalés dans l'éclosion en cours aux Pays-Bas, dont 182 hospitalisations et 1 décès; $94 \%$ des cas avaient été vaccinés (3). L'organisme Fraser Health a déclaré une éclosion de rougeole dans un groupe qui s'oppose à la vaccination pour des raisons religieuses le 8 mars 2014 et, en date du 8 avril 2014, avait recensé 375 cas, dont deux hospitalisations (9). Ces éclosions dans des populations semblables, mais vaccinées montrent avec quelle rapidité les cas de rougeole peuvent se transformer en éclosion généralisée et mettent en relief l'importance de la préparation en amont et de la mise en œuvre immédiate des mesures associées.

Cette épidémie de rougeole en Alberta met en évidence l'importance de la vaccination systématique des enfants, car tous les cas étaient non vaccinés. Les contacts familiaux qui avaient signalé des antécédents de la maladie ou indiqué avoir reçu une dose de vaccin contenant le virus de la rougeole n'ont pas été infectés. II existe plusieurs obstacles à la réalisation d'une vaste couverture vaccinale dans la population, comme le faible statut socioéconomique (10), la difficulté d'accès aux services (10-12) et le manque de connaissances ou la désinformation à propos de la vaccination (11-13). Dans cette population, les convictions religieuses et les normes culturelles ont été les principaux obstacles. Il est important de reconnaître les obstacles propres à une collectivité et de travailler avec elle à les surmonter. Au cours de cette éclosion, l'établissement d'une relation de confiance et de respect entre les services de santé publique locaux et les collectivités a mené à un effort concerté afin de réduire les risques de transmission dans la population à plus grande échelle.

Depuis la fin de l'éclosion, le travail de préparation se poursuit dans l'éventualité d'une prochaine éclosion de rougeole. Un plan exhaustif à l'échelle de la province est en élaboration et comprend un certain nombre de mesures et d'actions dont les principales sont : l'évaluation des cas, le diagnostic, la prévention et le contrôle des infections, les mesures de santé publique et la vaccination. En outre, une enquête a été menée dans les cinq zones sanitaires en Alberta en vue de déterminer les interactions entre les collectivités qui ne se font pas vacciner. Les données sont actuellement analysées et serviront à voir comment les maladies évitables par la vaccination risquent de se propager sur le territoire de la province.

\section{Conclusion}

Cette éclosion de rougeole met en évidence l'importance de la compréhension de la nature démographique des collectivités locales, de la surveillance continue des taux de couverture vaccinale et de la collaboration avec les intervenants internes et externes. Bien qu'il existe de nombreux facteurs pouvant contribuer à l'ampleur et à la gravité d'une éclosion, la planification et la relation avec la collectivité ont été les éléments clés qui ont permis de limiter cette éclosion à 10 ménages. L'exploration de stratégies visant à accroître la couverture vaccinale des populations non vaccinées est cruciale pour prévenir de futures éclosions de rougeole. Lorsque l'acceptation de la vaccination n'est pas uniforme, d'autres stratégies de santé publique devraient être planifiées et mises en 
œuvre afin de prévenir une propagation plus importante de la rougeole, par exemple, la détermination des interactions entre les collectivités en vue d'anticiper la propagation et l'établissement de relations avec les collectivités afin de mieux mettre en œuvre les mesures de prévention et de contrôle des infections en cas d'éclosion.

\section{Références}

(1) Comité consultatif national de l'immunisation. Vaccins actifs (partie 4).Guide canadien d'immunisation [Internet]. Ottawa: Agence de la santé publique du Canada, 2012. http://www.phac-aspc.gc.ca/publicat/ciggci/p04-meas-roug-fra.php.

(2) National Center for Immunization and Respiratory Diseases. Measles. Atlanta: Centers for Disease Control and Prevention; 2009 [consulté le 10 mars 2014].http://www.cdc.gov/measles/about/index.html

(3) National Institute for Public Health and the Environment. Mazelenepidemie 2013-2014. The Netherlands: Ministry of Health, Welfare and Sport.

Accès : http://www.rivm.nl/Onderwerpen/M/Mazelen/Mazelenepidemie_2013_2014

(4) Knol MJ, Urbanus AT, Swart EM, Mollema L, Ruijs WL, van Binnendijk RS, et al. Large ongoing measles outbreak in a religious community in the Netherlands since May 2013. Euro Surveill 2013; 18(36).

(5) Alberta Health. Public Health Notifiable Disease Management Guidelines: Measles. Alberta: Government of Alberta; Nov 2013 [consulté le 10 mars 2014].http://www.health.alberta.ca/documents/GuidelinesMeasles-2013.pdf

(6) Public Health England. Measles cases in England: update to end-June 2013. Health Protection Weekly Report, Aug 9 2013; 7(32).

(7) Agence de la santé publique du Canada. Rougeole. Ottawa : Agence de la santé publique du Canada; 24 octobre 2013; http://www.phac-aspc.gc.ca/im/vpd-mev/measles-rougeole-fra.php

(8) Bureau de surveillance et de vigie. Rapport final de l'épidémie provinciale de rougeole survenue en 2011. Québec : Ministère de la Santé et des Services sociaux; 2012 http://www.msss.gouv.qc.ca/sujets/prob_sante/rougeole/portrait2011.php

(9) Fraser Health. Health Alert: Measles in Fraser East. Surrey, BC: Fraser Health; 2014; http://www.fraserhealth.ca/your_health/immunizations/measles/news-and-updates/

(10) Santoli J, Szilagyi P, Rodewald L. Barriers to immunization and missed opportunities. Pediatr Ann 1998; 27(6):366-374.

(11) Mills E, Jadad A, Ross C, Wilson K. Systematic review of qualitative studies exploring parental beliefs and attitudes toward childhood vaccination identifies common barriers to vaccination. J Clin Epidemiol 2005; 58:1081-1088.

(12) Lieber M, Colden F, Colon A. Childhood immunizations: A parental education and incentive program. J Pediatr Health Care 2003; 17:240-244.

(13) Evers D. Teaching mothers about childhood immunizations. Am J Matern Child Nurs 2001; 26(5):253-6.

\section{Remerciements}

Les auteurs tiennent à remercier le $D^{r}$ Kevin Fonseca du laboratoire provincial de la santé publique (ProvLab) pour son aide à la rédaction de la section des méthodes de laboratoire; Martin Lavoie, Dana Paquette et Heather Gagnon pour la révision du manuscrit; et tous les membres du personnel de l'Alberta Health Services, du ministère de la Santé de l'Alberta et du ProvLab qui ont travaillé avec diligence à la gestion de cette éclosion de rougeole. 


\section{Conflit d'intérêts}

II n'y a aucun conflit d'intérêts à déclarer.

\section{Financement}

Aucun financement externe n'a été reçu. 